\title{
Amplitude equation and long-range interactions in underwater sand ripples in one dimension
}

Schnipper, Teis; Mertens, Keith; Ellegaard, Clive; Bohr, Tomas

Published in:

Physical Review E

Link to article, DOI:

10.1103/PhysRevE.78.047301

Publication date:

2008

Document Version

Publisher's PDF, also known as Version of record

Link back to DTU Orbit

Citation (APA):

Schnipper, T., Mertens, K., Ellegaard, C., \& Bohr, T. (2008). Amplitude equation and long-range interactions in underwater sand ripples in one dimension. Physical Review E, 78(4), 047301.

https://doi.org/10.1103/PhysRevE.78.047301

\section{General rights}

Copyright and moral rights for the publications made accessible in the public portal are retained by the authors and/or other copyright owners and it is a condition of accessing publications that users recognise and abide by the legal requirements associated with these rights.

- Users may download and print one copy of any publication from the public portal for the purpose of private study or research.

- You may not further distribute the material or use it for any profit-making activity or commercial gain

- You may freely distribute the URL identifying the publication in the public portal 


\title{
Amplitude equation and long-range interactions in underwater sand ripples in one dimension
}

\author{
Teis Schnipper, ${ }^{1}$ Keith Mertens, ${ }^{2}$ Clive Ellegaard, ${ }^{3}$ and Tomas Bohr ${ }^{1}$ \\ ${ }^{1}$ Department of Physics and Center for Fluid Dynamics, The Technical University of Denmark, 2800 Kgs. Lyngby, Denmark \\ ${ }^{2}$ Department of Mathematics, Colorado State University, Fort Collins, Colorado 80523-1874, USA \\ ${ }^{3}$ The Niels Bohr Institute, Blegdamsvej 17, 2100 Copenhagen Ø, Denmark
}

(Received 14 February 2008; revised manuscript received 29 August 2008; published 10 October 2008)

\begin{abstract}
We present an amplitude equation for sand ripples under oscillatory flow in a situation where the sand is moving in a narrow channel and the height profile is practically one dimensional. The equation has the form $h_{t}=-\epsilon(h-\bar{h})+\left(\left(h_{x}\right)^{2}-1\right) h_{x x}-h_{x x x x}+\delta\left(\left(h_{x}\right)^{2}\right)_{x x}$ which, due to the first term, is neither completely local (it has long-range coupling through the average height $\bar{h})$ nor has local sand conservation. We argue that this is reasonable and show that the equation compares well with experimental observations in narrow channels. We focus in particular on the so-called doubling transition, a secondary instability caused by the sudden decrease in the amplitude of the water motion, leading to the appearance of a new ripple in each trough. This transition is well reproduced for sufficiently large $\delta$ (asymmetry between trough and crest). We finally present surprising experimental results showing that long-range coupling is indeed seen in the initial details of the doubling transition, where in fact two small ripples are initially formed, followed by global symmetry breaking removing one of them.
\end{abstract}

DOI: 10.1103/PhysRevE.78.047301

PACS number(s): 47.54.-r, 45.70.Qj, 47.20.Lz

Sand ripples under water waves near the coast display a rich repertoire of patterns and interesting transitions among them. These structures have been investigated theoretically and in laboratory experiments for many years starting with the pioneering work of Ayrton and Bagnold $[1,2]$. The phenomenology is in many ways similar to classical pattern forming systems like Rayleigh-Bénard convection, but in contrast to the latter, an amplitude equation [3] has never been derived for the periodically driven sand ripples. In the present paper we propose a candidate for such an amplitude equation, so far restricted to one spatial dimension, i.e., systems of parallel ripples. We hope thereby to be able to rationalize some of the many interesting classical and recent observations on the pattern forming properties of such systems [4-7].

An oscillatory flow above a layer of sand leads to the formation of ripples if the flow is strong enough to set the sand in motion. The initial ripples are small and often called "rolling grain ripples" [2]. As time progresses they coarsen and finally turn into stable "vortex ripples." Presumably the coarsening is logarithmic [6] although the precise evolution to fully developed vortex ripples depends on the initial preparation of the sand. The formation of ripples can either be seen as an intrinsic instability of a flat bed to oscillatory flow, leading to a wavy bottom [8], or as the tendency for loose grains to lump together [9]. This process can occur whenever the flow is able to tear sand grains loose from the bed, and this happens when the so-called Shields parameter $\sigma$, the nondimensional shear stress, exceeds a threshold value $\sigma_{c}[10]$. In any case, the flat bed is unstable to a band of wavelengths, and the fully developed system typically has a wavelength much larger than the one of the initial instability. This final wavelength is set by the external flow: It is roughly proportional to the amplitude of the water motion, and independent of the frequency [11]. Changes in frequency influence the shape of the ripples and can lead to secondary instabilities [7]. The maximal slope of the fully developed ripples is close to the angle of repose for wet sand [4], in our case around $30^{\circ}$.

The final wavelength selection is governed by the separation vortex appearing in the trough on the lee side of the ripple crest, whose maximal size is set by the amplitude of the water motion near the bottom. The separation process is also what makes the hydrodynamics of sand ripples so difficult, since the only simple model for such flows (the boundary layer approximation) becomes singular at separation points. Currently no simple model exists which can predict separated flow along an arbitrary height profile (short of solving the full Navier-Stokes equations). When one adds to this the fact that the flow is turbulent and the complications of the granular dynamics, we are far from a model for the dynamics that would make it possible to derive an amplitude equation from "first principles." And even with such a model, the problem in understanding the ripple structures is one of keeping track of minute changes over long times, resulting from differences of sizable, almost opposite, changes occurring in each half-stroke. The best model currently available $[12,13]$ does this by assigning to the ripples a transport function, which predicts the amount of transport in each half-stroke, depending on the ripple size. This model has been quite successful in predicting certain properties of the ripple patterns, but relies on the representation of a height profile in terms of elementary ripples making, e.g., the creation of new ripples somewhat artificial.

In our amplitude equation, the periodic forcing will not appear explicitly. We are interested in the long time behavior, in the sense that we shall try to reproduce experimental observation made on time scales that contain many periods. The height function $h(x, t)$ appearing in the amplitude equation is thus equivalent to the height of the real sand-layer time-averaged over one period of the drive. To derive an amplitude equation for $h$, we shall rely on symmetry properties and the assumption that terms with lower derivatives and powers of the field will dominate. The simplest candidate, 


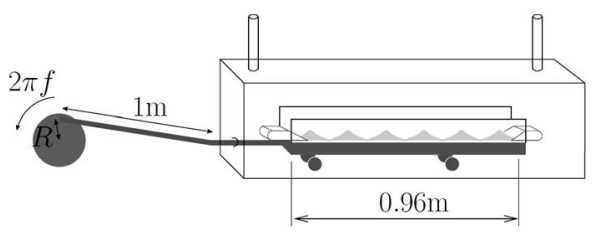

FIG. 1. The experimental setup: A narrow channel with a layer of sand is moved periodically in a plexiglass tank.

which has been discussed before in the context of sand ripples [14] and more generally $[15,16]$, is

$$
h_{t}=A\left(\left(h_{x}\right)^{2}-\theta\right) h_{x x}-\nu h_{x x x x}+B\left(\left(h_{x}\right)^{2}\right)_{x x} .
$$

The first term (proportional to $A$ ) on the right-hand side (RHS) generates a flat-bed instability as in the KuromotoSivashinsky equation [3], when the nondimensional parameter $\theta$ is positive. This parameter is thus analogous to $\left(\sigma-\sigma_{c}\right)$. For positive $\theta$ this instability remains as long as $\left|h_{x}\right|<\sqrt{\theta}$, roughly the angle of repose.

The term proportional to $\nu$ provides the necessary damping at small scales in the unstable case. The last term breaks the up-down symmetry $h \rightarrow-h$. This is important for the secondary instabilities, since they distinguish between crest and trough of the ripples. The field $h$ is locally conserved in the sense that $h_{t}=-J_{x}$, where the surface current is

$$
J=-A\left(\frac{1}{3}\left(h_{x}\right)^{3}-\theta h_{x}\right)-\nu h_{x x x}-B\left(\left(h_{x}\right)^{2}\right)_{x} .
$$

The problem with (1) is, that it displays coarsening, which goes on until the system contains only one large ripple $[15,16]$. This behavior is typical of systems with a local conservation law [15], and since sand is of course conserved in the dynamics, it seems obvious that the amplitude equation must have this property. Actually, this is not so clear, since an amplitude equation written entirely in terms of the local sand height leaves out a large number of degrees of freedom. The simplest way to quench the instability at large length scale is to add a linear term $-a(h-\bar{h})$ to the RHS of (1) - as in the Swift-Hohenberg equation [3]. This violates the local conservation property, but the explicit appearance of the average height $\bar{h}=L^{-1} \int_{0}^{L} h d x$ ensures that it satisfies the global conservation law $\frac{d \bar{h}}{d t}=0$ (for periodic boundary conditions). This linear term thus breaks two standard assumptions: Local conservation and local coupling. Both of these violations are reasonable. It is well known that sand is not simply transported along the surface. In fact sand moves into the ripples at some places and resurfaces at other places [17], and it is also thrown into the water current at some places and returned to the interface at others. Even though sand is of course locally conserved, we cannot expect the sand current to be simply a function of the local height. Also, since the hydrodynamics driving the grains is incompressible and generates vortices we should expect long-distance effects [19].

Note that in the case of ripples generated by DC flow a much more detailed representation of the dynamics has been developed in terms of saltation and reptation currents (see, e.g., [18]). In the oscillatory case, as discussed above, the location and strength of the separation vortices is the main ingredient, and since we do not know how to handle this accurately we simply assume that the finite length of separation bubbles (for a given water amplitude) can be translated to a finite time scale $a^{-1}$ beyond which the ripple forming instability is damped.

By appropriate rescaling [20] the amplitude equation can now (assuming that $\theta>0$ ) be brought on dimensionless form

$$
h_{t}=-\epsilon(h-\bar{h})+\left(\left(h_{x}\right)^{2}-1\right) h_{x x}-h_{x x x x}+\delta\left(\left(h_{x}\right)^{2}\right)_{x x}
$$

with only two parameters, $\epsilon=a \nu A^{-2} \theta^{-2}$ and $\delta=B \nu^{-1 / 2} A^{-1 / 2}$. For arbitrary choice of $\epsilon$ and $\delta$ the dynamics described by (3) seems to converge to a stationary state. This is guaranteed if the dynamics is potential [3], i.e., of the form $h_{t}=-\frac{\delta}{\delta h} \mathcal{H}\left\{h, h_{x}, h_{x x}, \ldots\right\}$. For $\delta=0$, a potential indeed exists and is of the form

$$
\mathcal{H}=\frac{1}{2} \int_{0}^{L}\left(\epsilon(h-\bar{h})^{2}-\left(h_{x}\right)^{2}+\frac{1}{6}\left(h_{x}\right)^{4}+\left(h_{x x}\right)^{2}\right) d x
$$

but for $\delta \neq 0$ this is no longer true, so in principle the asymptotic dynamics could be more complicated.

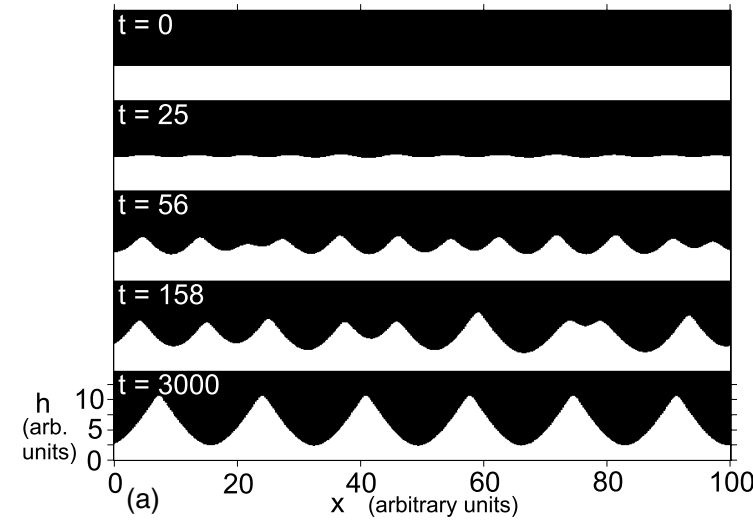

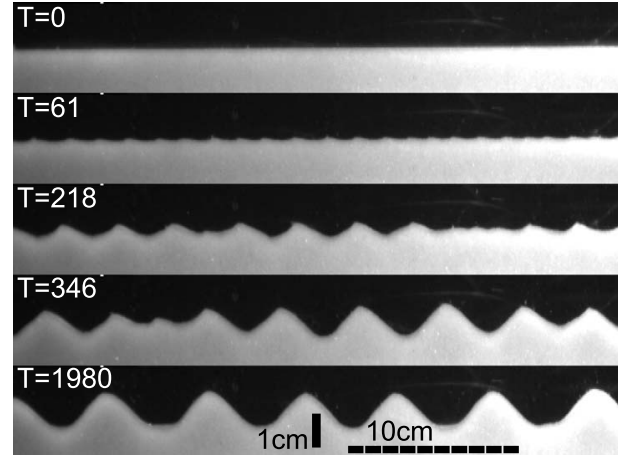

(b)

FIG. 2. Temporal development of flat bed instability. (a) For the amplitude equation (3), $\epsilon=0.03$ and $\delta=1$ with periodic boundary conditions. (b) In the experiment [side view of the central region $(36 \mathrm{~cm})$ of the channel]. The length of each black bar is $1 \mathrm{~cm}-$ note that the vertical scale is twice that of the horizontal. $T$ denotes the number of oscillations. 
The predictions of the amplitude equation (3) will be compared with experimental observations obtained by oscillating a tray of sand in a narrow channel. The experimental setup is seen in Fig. 1. A sledge with a narrow channel of width $11 \mathrm{~mm}$ is moved using an eccentric coupling to a rotating wheel. The sledge moves on rails inside a transparent plexiglass tank fixed in the laboratory frame. The tank is filled with tap water such that there is no free surface except in the two vertical pipes that compensate volume changes as the driving rod penetrates the tank. Adjustable driving parameters are the sledge amplitude $R$ and frequency $f$, typical values are $R=30 \mathrm{~mm}$ and $f=1 \mathrm{~Hz}$. As long as $R \ll 1 \mathrm{~m}$ (length of the arm) the motion is nearly harmonic. In Fig. 2(a) we show a sequence of height profiles obtained by numerical solution of the amplitude equation (3) for $\delta=1$ starting with a flat bed. For comparison, Fig. 2(b) shows snapshots of the development in the experimental system. The initial instability from a flat bed occurs in the model around the most unstable wave number $k=1 / \sqrt{2}$ and subsequently the wavelength increases by coarsening. For $\epsilon=0$ this would lead to a ripple size growing logarithmically, i.e., $\lambda(t) \sim \ln t$ [15]. For $\epsilon>0$ the process stops at a finite wavelength as we expect. Figure 3 shows the number of ripples $N=L k / 2 \pi$ in a system of size $L=200$, as function of $\epsilon$ for various values of $\delta$ always computed by starting from a flat bed with a slight randomness. As can be seen from the data, $N$ seems to follow a power law $N(\epsilon) \sim \epsilon^{\alpha}$, but the exponent seems to depend on $\delta$, going from $\alpha \approx 0.12$ for $\delta=0$ to $\alpha \approx 0.5$ for $\delta=5$. The value $\alpha=0.5$ corresponds to the scaling of the lower edge of the unstable wave-number band for a flat bed, i.e., $\left(k_{e}\right)^{2}=0.5\left[1-(1-4 \epsilon)^{1 / 2}\right] \approx \epsilon$, for $\epsilon \ll 1$. We do not know why this "trivial" scaling is apparently found only for large $\delta$. As we shall see later, a nonzero $\delta$ is necessary to understand the "doubling transition" and thus we can, in the physically relevant case of not too small $\delta$, roughly identify the amplitude of the water motion (which determines the ripple wavelength) with $\epsilon^{-1 / 2}$. The experimental control parameter most obviously related to $\delta$ is the frequency $f$, which does not

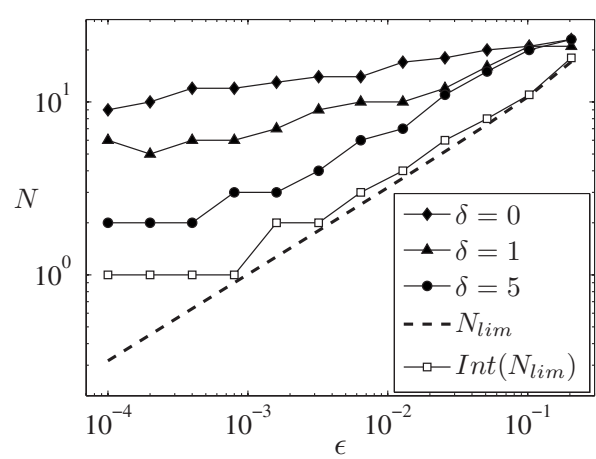

FIG. 3. Number of ripples in a domain of size $L=200$ for $\delta=0$, 1 , and 5 as function of $\epsilon$. Also shown (dashed line) is the corresponding "limiting number," $N_{\lim }=L k_{e}(\epsilon) / 2 \pi$ and the nearest integer value, $\operatorname{Int}\left(N_{\text {lim }}\right)$, larger than $N_{\text {lim }}$ (full line with $\square$ ). The numerics were done with finite difference methods using a variable-step Runge-Kutta algorithm (MATLAB) for time integrations. Periodic boundary conditions were used, and for these long simulations (around $10^{6}$ time steps) it was important to use the conserving form (2) for all terms except the one proportional to $\epsilon$ to avoid drift of the mean height.

have much influence on the wavelength, but changes the ripple shapes.

One should note that the determination of $N(\epsilon)$ is difficult numerically due to the large equilibration times and the inherent nonuniqueness of the final state, which is also a property of the experiment [2]. We do not have sufficient statistics to give meaningful error bars in Fig. 3, but different runs can give ripples numbers $N$ differing by around $20 \%$. One can force this variation in $N$ further by starting in a "wrong" stationary state. As an example, we have found a stationary state for $L=200, \delta=1$, and $\epsilon=0.025$ with $N=13$ ripples. Taking this state as initial condition we can vary $\epsilon$ within the whole interval $[0.009,0.08]$ without changing $N$. The same is true for the experiments: Around each state there is a "stability balloon" inside which no bifurcations take place even though parameters are changed. Note however that in the

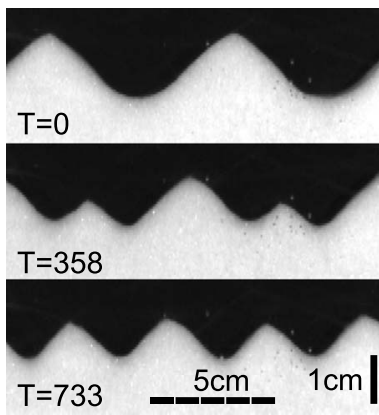

(a)

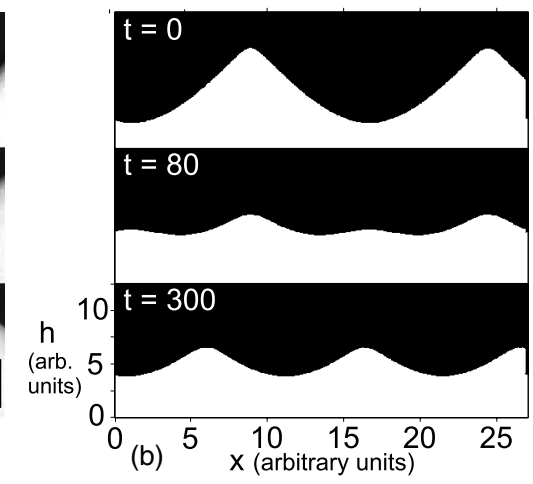

(b)

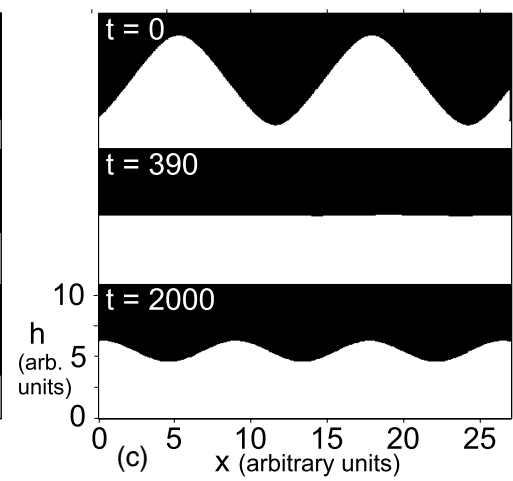

(c) 5 (arbitrary units)

FIG. 4. (a) "Doubling" transition by approximately halving the stroke length from initially $90 \mathrm{~mm}$ (top). After some time a new ripple appears in each trough leading to an intermediate state with twice as many ripples, which may then coarsen to fewer ripples in the final state. The initial doubling of the ripple number happens generically going to a state with more ripples. Note the slight drift typical of systems with a spontaneously broken translational symmetry. Each black bar is $1 \mathrm{~cm}$ long. (b) Doubling transition of (3) when changing $\epsilon$ from 0.025 to 0.1 for $\delta=1$. (c) Transition when changing $\epsilon$ from 0.006 to 0.2 for $\delta=0$. When $\delta=0$ the profile is up-down symmetric and doubling cannot occur. Instead, the amplitude initially decays almost to zero whereupon a modulation of the amplitude sets in. The decaying peaks or troughs subsequently split leading to an increased number of ripples in the final state. In all cases, we only show part of the system with a few ripples to make the bifurcation scenario clear. 
experiments the boundary conditions are not periodic, and ripples can move across the outer boundary allowing the system to adjust the wavelength slightly. Beyond these boundaries three distinct types of transitions have been described [7]. The "pearling" transition, where the frequency is suddenly increased, leads to a new pattern, where a checkerboard of small ripples ("pearls") appear in the troughs of the old ones. In the "bulging" transition the amplitude of the water (tray) motion is suddenly increased and the ensuing coarsening process leads through a checkerboard pattern of dilations and compressions. Finally, a sudden decrease in amplitude introduces new ripples via "doubling," whereby a new ripple emerges in each trough. This is true even if the number of ripples in the final pattern is substantially less than 2 times the original one-the superfluous ripples are subsequently removed by "bulging." In the following we shall concentrate on the doubling since it is an essentially one-dimensional (1D) transition. Figure 4(a) shows snapshots from an experimentally observed doubling transition in our setup. Time increases downward and in the middle panel a new ripple is apparent in each trough. In Fig. 4(b) similar sequences are generated numerically from (3) when $\epsilon$ is changed from 0.025 to 0.13 with (fixed) $\delta=1$. The threshold for doubling in this state is $\epsilon=0.08$. For this to occur, it is crucial that $\delta>0$. For $\delta=0$ [Fig. 4(c)] there is no difference between crests and troughs and the bifurcation typically proceeds by "peak-splitting" (either around crests or troughs) and this typically occurs after a substantial decay of the amplitude [Fig. 4(c) at $t=390$ ].

In Fig. 5 we present a sequence of experimentally generated height profiles showing the doubling transition from Fig. 4(a) in more detail. It is seen that the initial instability actually leads to the birth of two new ripples in each trough. Very quickly (after a few oscillation periods), though, one of them wins and grows while the other decays. Surprisingly enough, the one that wins was in all experiments observed to

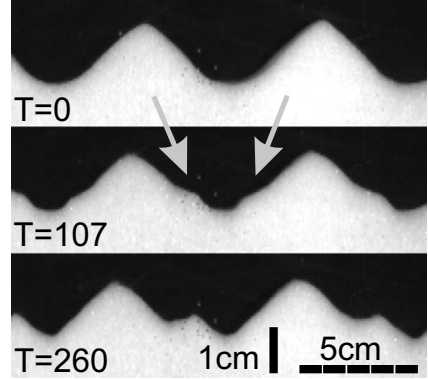

FIG. 5. Initial development of the doubling transition in Fig. 4(a). Initially, two ripples appear in all troughs, but only one grows at the expense of the other. Either all the right ones or the left ones.

be the same in all of the troughs - either all the left hand ones or all the right hand ones. In our experiment both cases occur, so we believe that this phenomenon is due to a genuine hydrodynamical coupling, not to asymmetry of our experiment. This effect is not captured by our amplitude equation, but it does illustrate that long-range coupling occurs. To examine this further, we repeated the experiment and stopped the oscillation when the system had clearly selected in which side the new ripples should grow. We then manually moved one ripple to the other side of the trough. Upon restarting the oscillation this ripple was moved back by the flow, clearly showing the strength of the hydrodynamical coupling. The fact that the new ripples initially form close to the reconnection point of the separation bubble shows again clearly that a more refined theory needs to include a model of separation.

We hope in future work to be able to understand the interesting three-dimensional features (as seen, e.g., in the "bulging transition" [7] or applying a skewed drive [21]) by extension of our model to two spatial dimensions.

We are grateful to Joachim Krug, Vincent Hakim, and Joachim Kruithof for helpful discussions.
[1] H. Ayrton, Proc. R. Soc. London, Ser. A 84, 285 (1910).

[2] R. A. Bagnold, Proc. R. Soc. London, Ser. A 187, 1 (1946).

[3] M. Cross and P. C. Hohenberg, Rev. Mod. Phys. 65, 851 (1993).

[4] A. Stegner and J. E. Wesfreid, Phys. Rev. E 60, R3487 (1999).

[5] M. A. Scherer, F. Melo, and M. Marder, Phys. Fluids 11, 58 (1999).

[6] G. Rousseaux, A. Stegner, and J. E. Wesfreid, Phys. Rev. E 69, 031307 (2004).

[7] J. L. Hansen, M. van Hecke, C. Ellegaard, K. H. Andersen, T. Bohr, A. Haaning, and T. Sams, Nature (London) 410, 324 (2001); Phys. Rev. Lett. 87, 204301 (2001).

[8] P. Blondeaux, J. Fluid Mech. 218, 1 (1990).

[9] K. H. Andersen, Phys. Fluids 13, 58 (2001).

[10] J. Fredsøe and R. Deigaard, Mechanics of Coastal Sediment Transport (World Scientific, Singapore, 1992).

[11] P. Nielsen, J. Geophys. Res. 86, 6467 (1981).

[12] K. H. Andersen, M.-L. Chabanol, and M. van Hecke, Phys.
Rev. E 63, 066308 (2001).

[13] K. H. Andersen, M. Abel, J. Krug, C. Ellegaard, L. R. Søndergaard, and J. Udesen, Phys. Rev. Lett. 88, 234302 (2002).

[14] J. Krug, Complex Syst. 4, 353 (2001).

[15] P. Politi, Phys. Rev. E 58, 281 (1998); 73, 036133 (2006).

[16] Z. Csahók, C. Misbah, F. Rioual, and A. Valance, Eur. Phys. J. E 3, 71 (2000).

[17] G. Rousseaux, H. Caps, and J.-E. Wesfried, Eur. Phys. J. E 13, 213 (2004).

[18] L. Prigozhin, Phys. Rev. E 60, 729 (1999).

[19] In reality the nonlocal term in our equation should have a finite range. One might, e.g., replace $\bar{h}$ by $L^{-1} \int_{0}^{L} h\left(x^{\prime}\right) e^{-\beta\left|x^{\prime}-x\right|} d x^{\prime}$. As seen later, we have coupling through our entire experimental channel, and thus the length scale $\beta^{-1}$ can be taken as infinite.

[20] $h \rightarrow \nu^{1 / 2} A^{-1 / 2} h, x \rightarrow \theta^{-1 / 2} \nu^{1 / 2} A^{-1 / 2} x, t \rightarrow \nu A^{-2} \theta^{-2} t$.

[21] F. Bundgaard, C. Ellegaard, K. Scheibye-Knudsen, T. Bohr, and T. Sams, Phys. Rev. E 70, 066207 (2004). 